\title{
Design of Cascode-Based Transconductance Amplifiers with Low-Gain PVT Variability and Gain Enhancement Using a Body-Biasing Technique
}

\author{
Nuno Pereira ${ }^{2}$, Luis B. Oliveira ${ }^{1,2}$, and João Goes ${ }^{1,2}$ \\ ${ }^{1}$ Centre for Technologies and Systems (CTS) - UNINOVA \\ ${ }^{2}$ Dept. of Electrical Engineering (DEE), Universidade Nova de Lisboa (UNL) \\ Campus FCT/UNL, 2829-516, Caparica, Portugal \\ nrf.pereira@gmail.com, l.oliveira@fct.unl.pt, jg@uninova.pt
}

\begin{abstract}
A body-biasing compensation scheme based on two proportional-toabsolute-temperature (PTAT) circuits is proposed to reduce the PVT variability of the DC gain of cascode amplifiers. A brief description of a basic PTAT is given as well as its application to cascode-based operational transconductance amplifiers. Simulation results show that the proposed compensated circuit amplifier exhibit a (DC) gain variability smaller than the original (uncompensated) circuit, while reaching a gain enhancement of about $3 \mathrm{~dB}$.
\end{abstract}

Keywords: amplifier, body-biasing, CMOS analog circuits, PVT compensation.

\section{Introduction}

In order to increase speed and reduce area, MOS devices are scaled down. However, this leads to short channel effects, thus reducing the intrinsic gain. Therefore, due to the supply reduction, high gain OpAmps are harder to design and cascode techniques are more difficult to employ [1]. In analog CMOS circuit design, the transistor is preferentially used in saturation. The intrinsic gain of a MOS transistor is given by

$$
A_{v i}=g_{m} / g_{d s}
$$

where $g_{m}$ is the transconductance and $g_{d s}$ is the output conductance. For a MOS device in saturation, assuming an approximate square law for the drain current, the transconductance is given by

$$
g_{m}=\mu C_{o x}^{\prime}(W / L)\left(V_{G S}-V_{t}\right)^{2}
$$

where $\mu$ is the carrier mobility, $C_{o x}^{\prime}$ is the oxide capacitance per unit of area, $W$ and $L$ are the width and length of the transistor, $V_{G S}$ is the biasing gate source voltage and $V_{t}$ is the threshold voltage. For a short channel, the increase of $V_{D S}$ leads to the decrease of $V_{t}$, thus increasing the drain current. This gives rise to an increase of $g_{d s}$. So, despite the increase of transconductance with scaling, the intrinsic gain is 
reduced [1]. This is illustrated in [2]. The temperature variation leads to a decrease in both the carrier mobility and the threshold voltage. In regards to the latter, it may decrease from $2 \mathrm{mV}$ up to $4 \mathrm{mV}$ for every $1^{\circ} \mathrm{C}$ rise [3]. These factors will have a direct effect on the transconductance of a transistor, as seen in (2). Also, process corners refer to the variation of fabrication parameters used in applying an integrated circuit design to a wafer. If a circuit running on devices fabricated at these process corners does not function as desired, the design is considered to have inadequate design margin. In this paper we present a circuit technique to reduce gain variability with temperature, supply and process variations in cascode amplifiers using a bodysuitable biasing circuitry and at the same time increase the overall amplifier gain by about $3 \mathrm{~dB}$.

\section{Relationship to Internet of Things}

Operational and transconductance amplifiers are, most probably, the most active building-block in analog and mixed-signal integrated circuits used in wireless and wire line communication systems. The work presented in this paper can contribute to the future development of the Internet of Things, since it can provide improved energy efficient circuit amplifier architectures, robust to temperature, supply and process variations. This ensures that a circuit manufactured to work, will perform as expected, regardless of the environment conditions.

\section{Cascode Amplifiers}

A single-stage operational transconductance amplifier (OTA) has usually a cascode configuration [4]. We consider a traditional cascode amplifier, shown in Fig. 1.

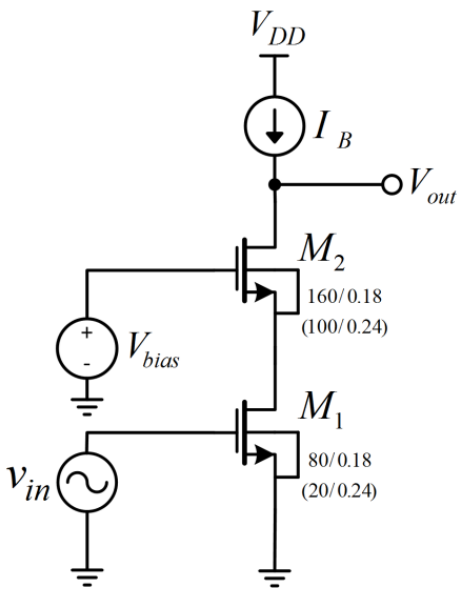

Fig. 1. Cascode Amplifier. All transistor sizes $(W / L)$ are in $\mu \mathrm{m}$. Sizes without brackets are for a $65 \mathrm{~nm}$ technology and those within brackets are for a $0.13 \mu \mathrm{m}$ one. 
The gain of this circuit is given by

$$
A_{v}=g_{m 1} \cdot\left[\frac{1}{g_{d s 1}} \cdot\left(\frac{g_{m 2}}{g_{d s 2}}\right)\right] \| R_{B}
$$

where $R_{B}$ is the resistance of the biasing current source, whose value is $100 \mu \mathrm{A}$ and $150 \mu \mathrm{A}$ for $65 \mathrm{~nm}$ and $130 \mathrm{~nm}$ technology, respectively. The positive power supply voltage is of $1.2 \mathrm{~V}$.

Fig. 2 shows the variations of $g_{m}, g_{d s}$, and gain of transistor $M_{2}\left(g_{m} / g_{d s}\right)$ for 65 $\mathrm{nm}$ and $130 \mathrm{~nm}$.

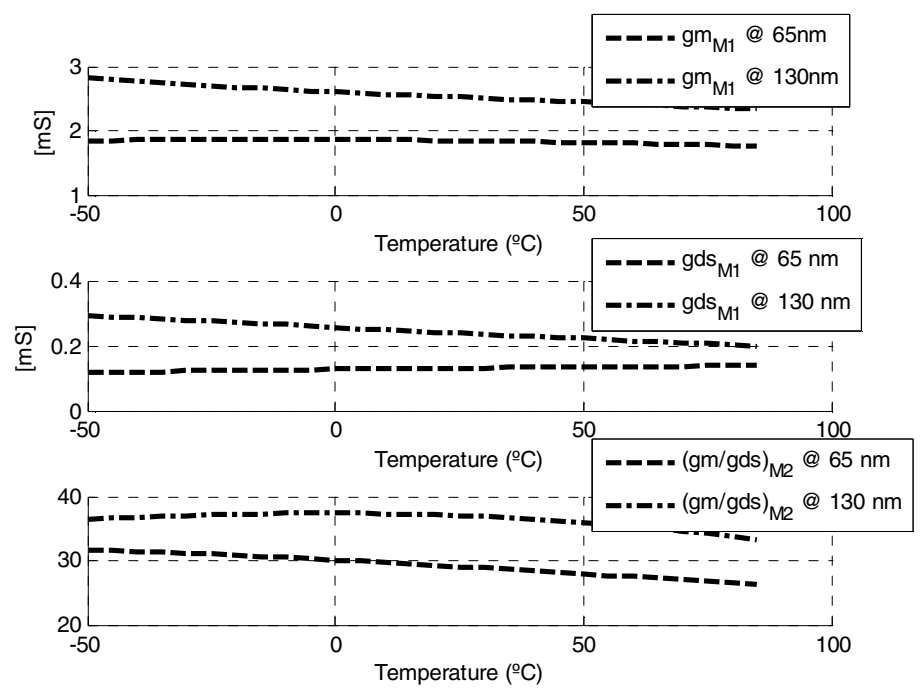

Fig. 2. Parameter variation with temperature for both node technologies

\section{$4 \quad$ Proposed Solution}

Fig. 3 presents the basic proportional-to-absolute-temperature (PTAT) circuit used in this paper, known as "constant transconductance" bias circuit, proposed in $[4,5]$. 


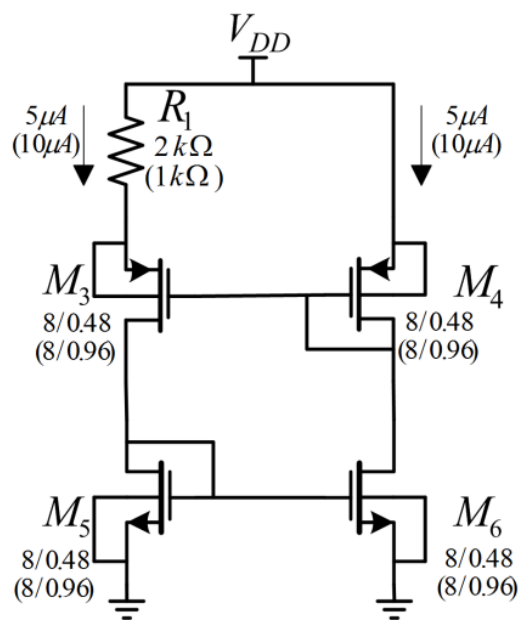

Fig. 3. Constant Transconductance Bias circuit. All transistor sizes $(W / L)$ are in $\mu \mathrm{m}$. Sizes without brackets are for a $65 \mathrm{~nm}$ technology and those within brackets are for a $0.13 \mu \mathrm{m}$ one. The supply voltage is of $1.2 \mathrm{~V}$.

We investigate the variation with temperature of the voltages $V_{A}, V_{B}$, and $V_{C}$, when all the transistors are matched.

The variation of $V_{A}$ is irrelevant, since it varies less than $5 \mathrm{mV}$ for the temperature range $\left(-50{ }^{\circ} \mathrm{C}\right.$ to $\left.+85^{\circ} \mathrm{C}\right)$. For both technology nodes, $V_{B}$ decreases with temperature while $V_{C}$ increases, as shown in Fig. 4.
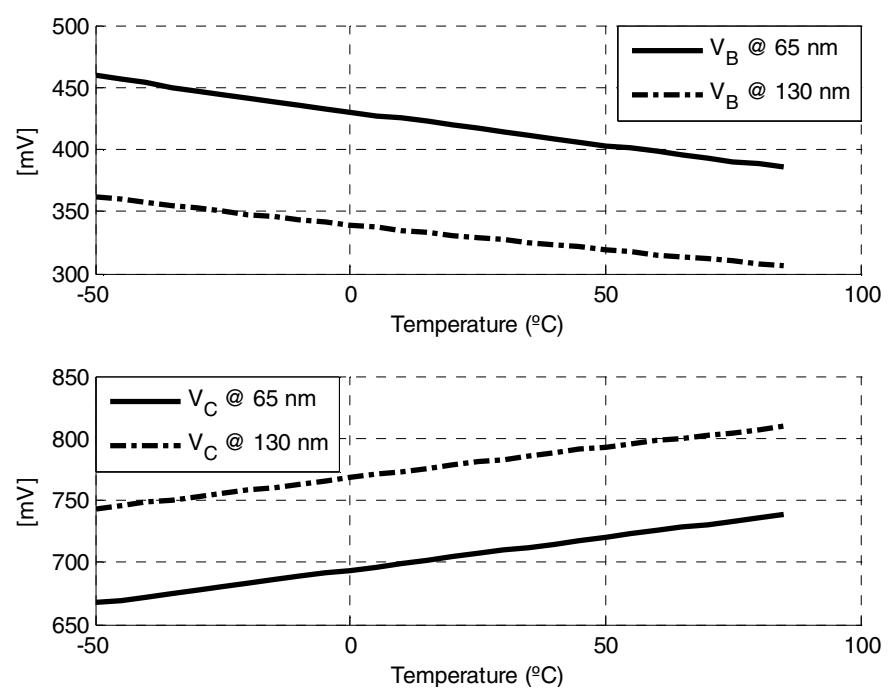

Fig. 4. Voltage variation at nodes $V_{B}$ and $V_{C}$ (for both node technologies) 
The cascode amplifier (Fig. 1) with body-biasing by the circuit of Fig. 3 is shown in Fig. 5. The $I_{B}$ current source is replaced with a current mirror, and every biasing voltage source is replaced with a MOSFET in diode configuration.

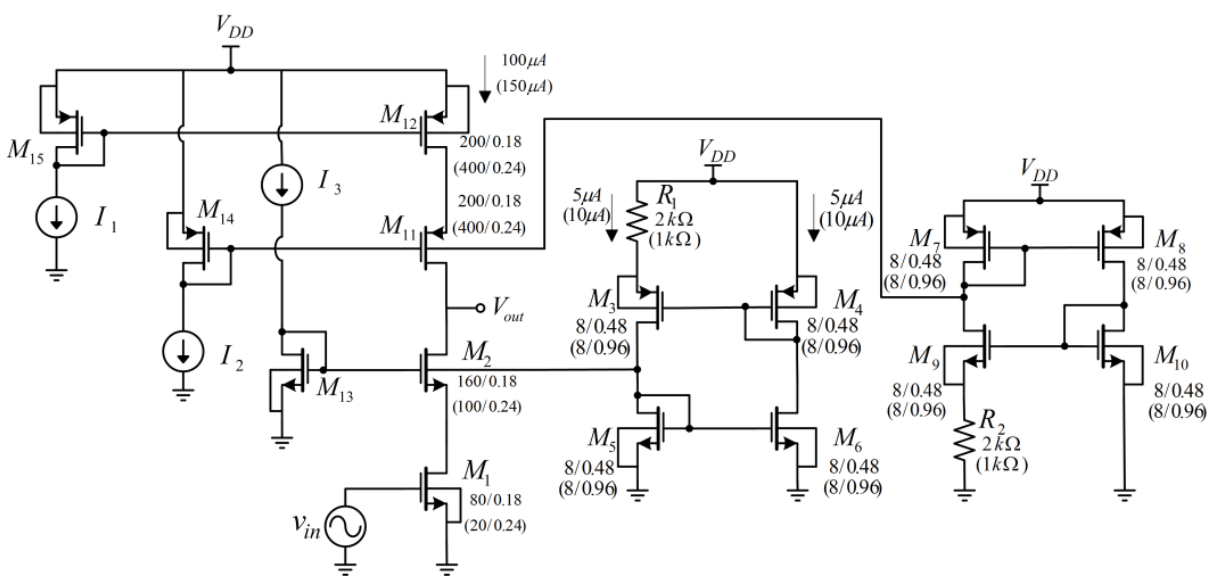

Fig. 5. Telescopic-cascode with body-biasing circuitry. All transistor sizes $(W / L)$ are in $\mu \mathrm{m}$. Sizes without brackets are for a $65 \mathrm{~nm}$ technology and those within brackets are for $0.13 \mu \mathrm{m}$.

In section 3 we have plotted the variations of $g_{m}$ and $g_{d s}$ in $65 \mathrm{~nm}$ and $130 \mathrm{~nm}$. The main objective is to choose one of these voltage variations and apply it to the bulk of the $M_{2}$ transistor of the cascode circuit, biasing it in order to reduce the variability of the intrinsic gain, thus narrowing the variability of the overall gain, and increase the total gain of the circuit. The voltage $V_{B}$ is used, since it decreases with temperature (for either technology), as seen in Fig. 4. If it is applied to the bulk of the $M_{2}$ transistor, it reduces the amplifier gain variability.

After replacing the current source with the current mirror, the MOSFETS that compose it also needed to have a temperature-independent behavior. Thus, the bulk of $M_{11}$ should be connected to a symmetrical voltage to that applied to the bulk of $M_{2}$. In order to do so, a mirrored version of the "constant transconductance" bias circuit was designed.

Fig. 6 presents the variations of the transconductances of the $M_{1,2}$ transistors, when using body-biasing compensation. As it can be seen, the intrinsic gain of the $M_{2}$ transistor has a lower variation than that obtained when no body-biasing technique is used (Fig. 2), for both node technologies. Furthermore, the $g_{d s}$ of the $M_{1}$ transistor also reduces its value while $g_{m}$ increases, thus boosting the gain. 


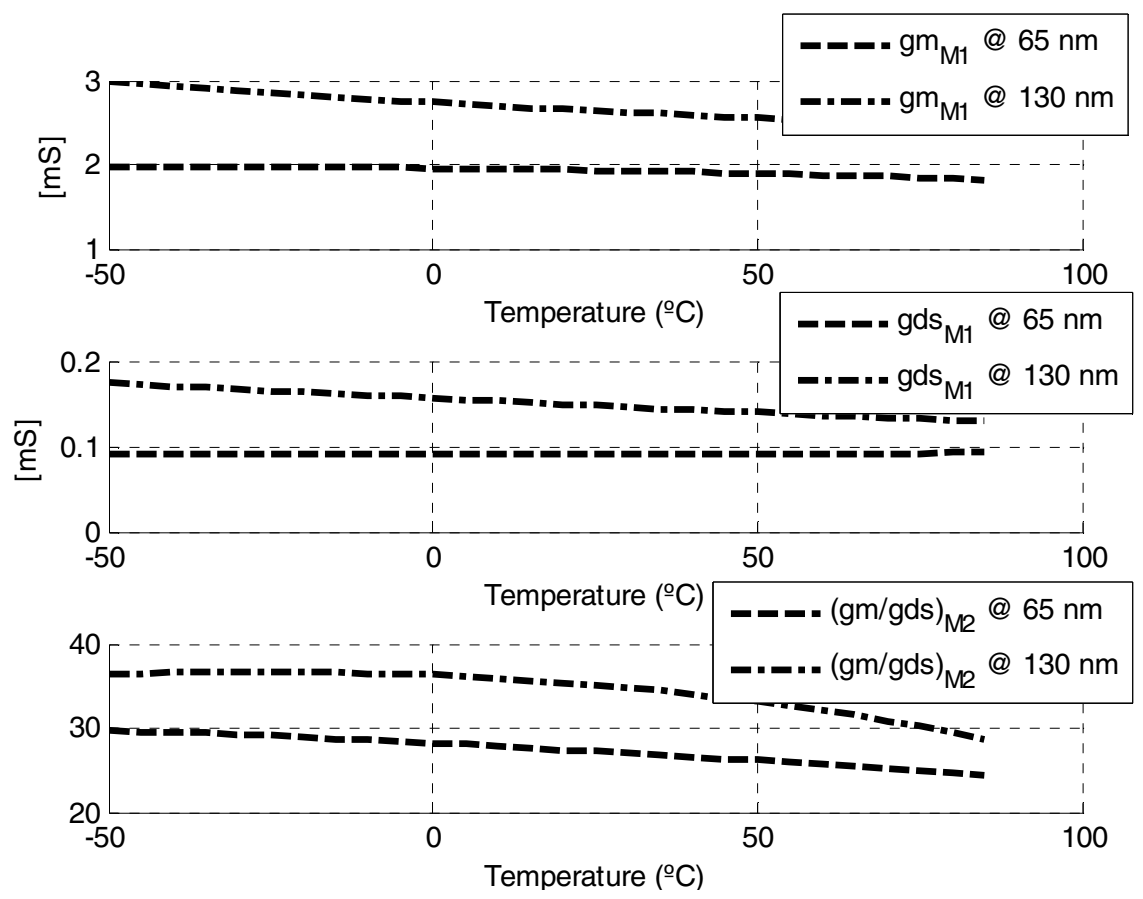

Fig. 6. Parameter variation with temperature with body-biasing compensation for both node technologies

\section{$5 \quad$ Simulations and Results}

All simulations are performed using BSIM3v3 models for standard logic $65 \mathrm{~nm}$ and $130 \mathrm{~nm}$ CMOS technology. A supply voltage of $1.2 \mathrm{~V}$ is used and the process variations considered are slow-slow (ss), typical-typical (tt) and fast-fast (ff). The temperature range is from -50 to $+85^{\circ} \mathrm{C}$.

In Fig. 7, the gain of the circuit is plotted, for both technology nodes, with and without body-biasing technique. For both technology nodes the gain variability is lower when using the body-biasing circuitry. Furthermore, there is an enhancing of the gain by about $3 \mathrm{~dB}$, as expected, as it was explained in section 4 .

With regard to supply variation, Fig. 8 shows that with a variation of $\pm 5 \%(1.14 \mathrm{~V}$ to $1.26 \mathrm{~V}$ ) of the supply voltage, the body-biasing circuitry leads to a lower variability for the gain (below $\pm 0.5 \mathrm{~dB}$ ). Some traces can be superimposed. 


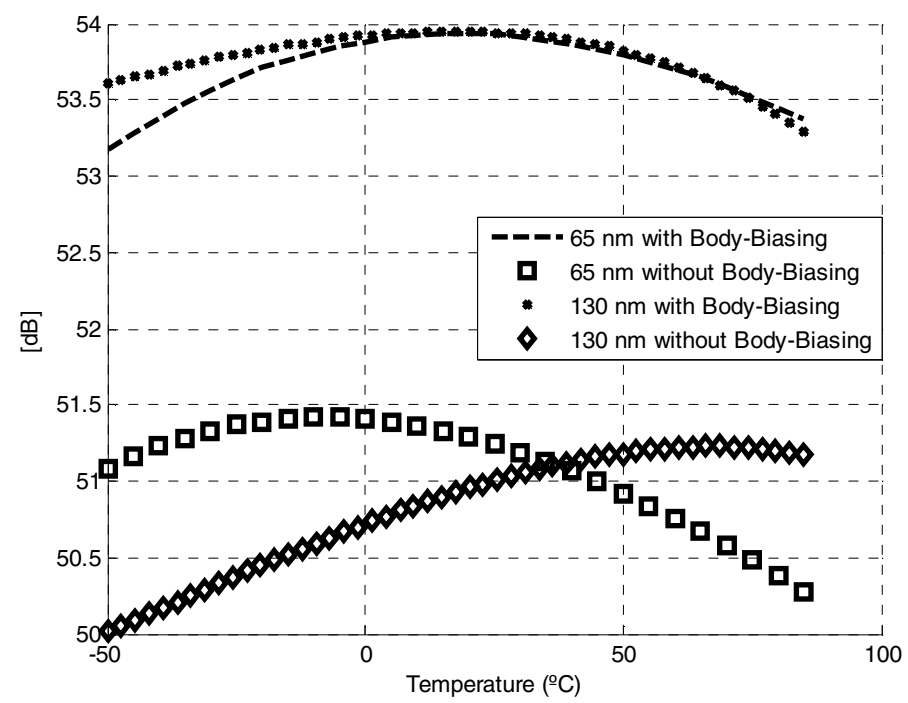

Fig. 7. Overall gain variability with temperature for both technologies node (with and without body-biasing technique)
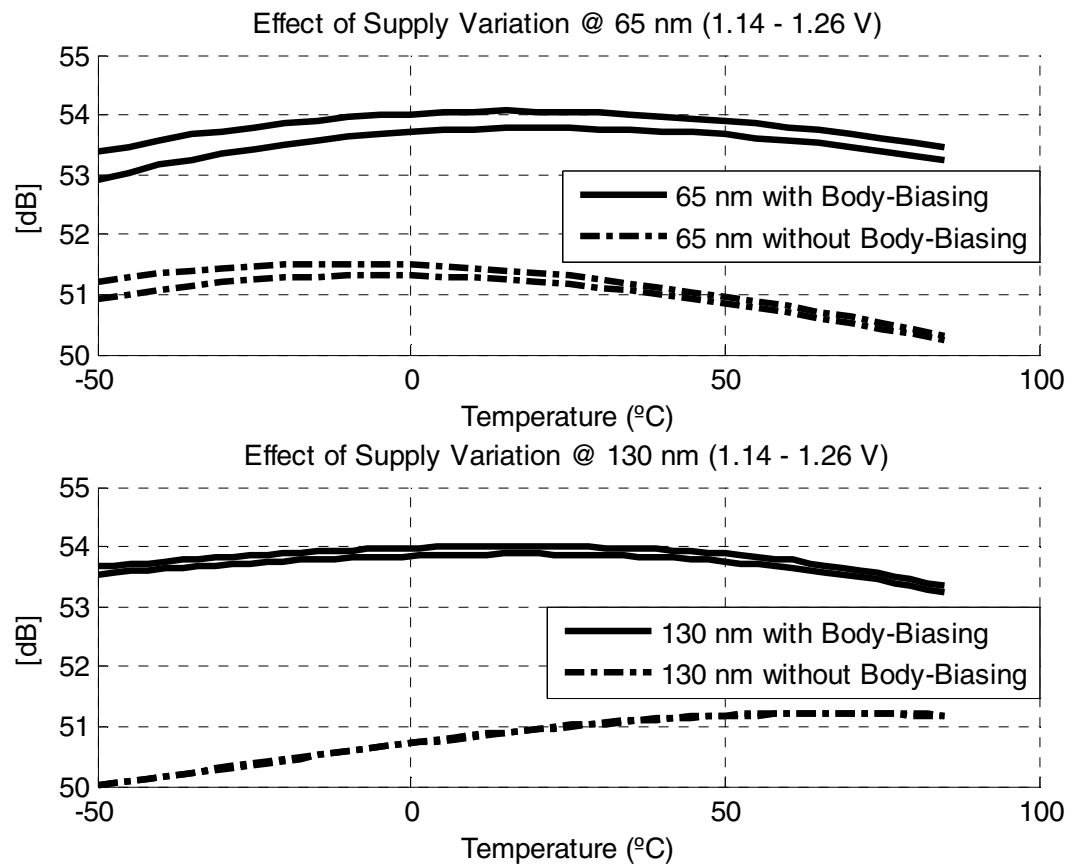

Fig. 8. Overall gain variability with temperature \& supply variation for both technologies node (with and without body-biasing technique) 
Concerning process variation, Fig. 9 and 10 show that for the processes considered (ss, $\mathrm{tt}$ and $\mathrm{ff}$ ), there is always an enhancement of the gain by about $3 \mathrm{~dB}$. For most of the cases, the (DC) gain variability is below $\pm 0.5 \mathrm{~dB}$ (except for process ff, at $130 \mathrm{~nm}$ node technology, where the variability is higher than that without body-biasing).

This body-biasing circuitry requires only an extra current consumption of about $5 \%$ to $7 \%$ (for both technologies) of the total current consumption of the amplifier. This results in low power consumption, for both node technologies, from a $1.2 \mathrm{~V}$ supply.
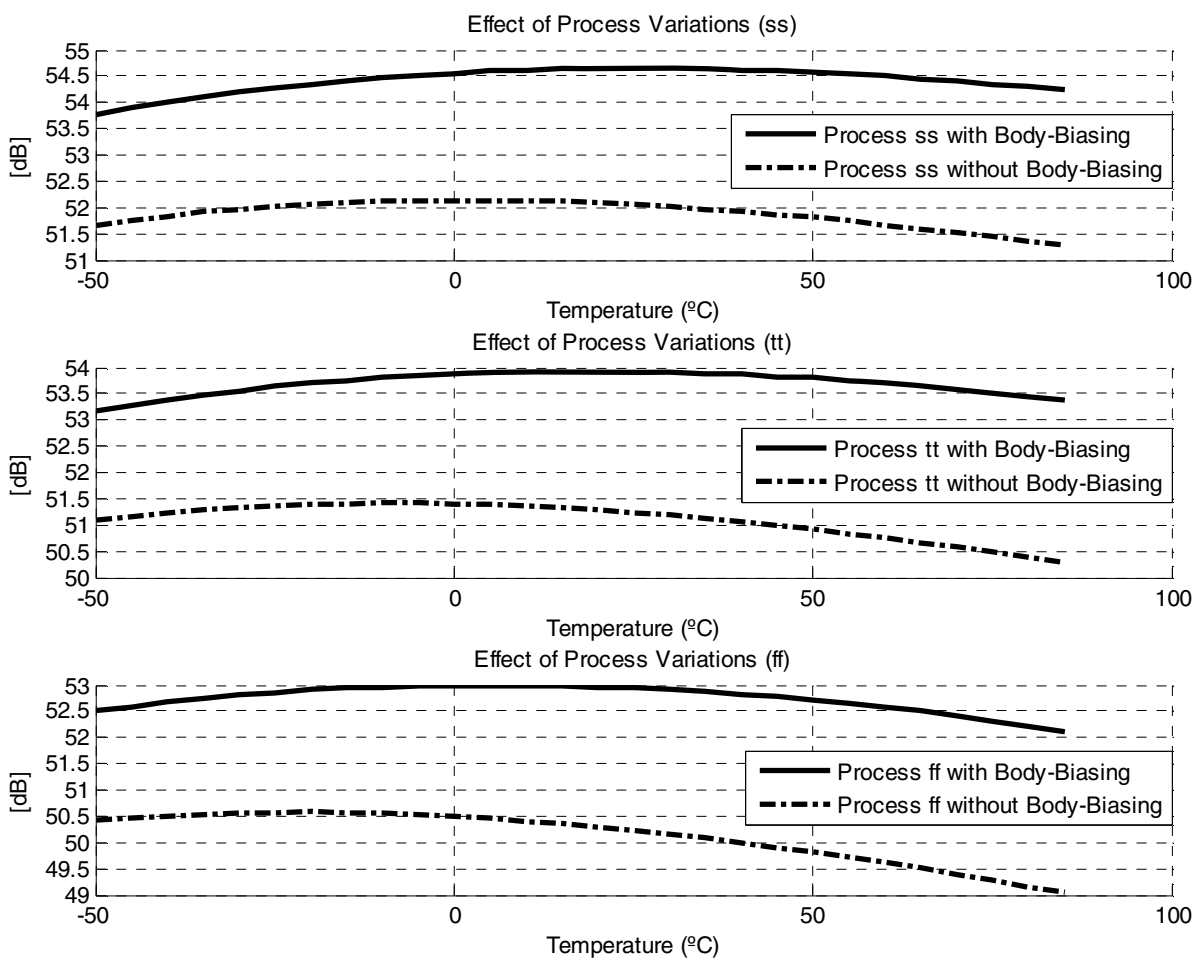

Fig. 9. Overall gain variability with temperature \& process variation for $65 \mathrm{~nm}$ (with and without body-biasing) 

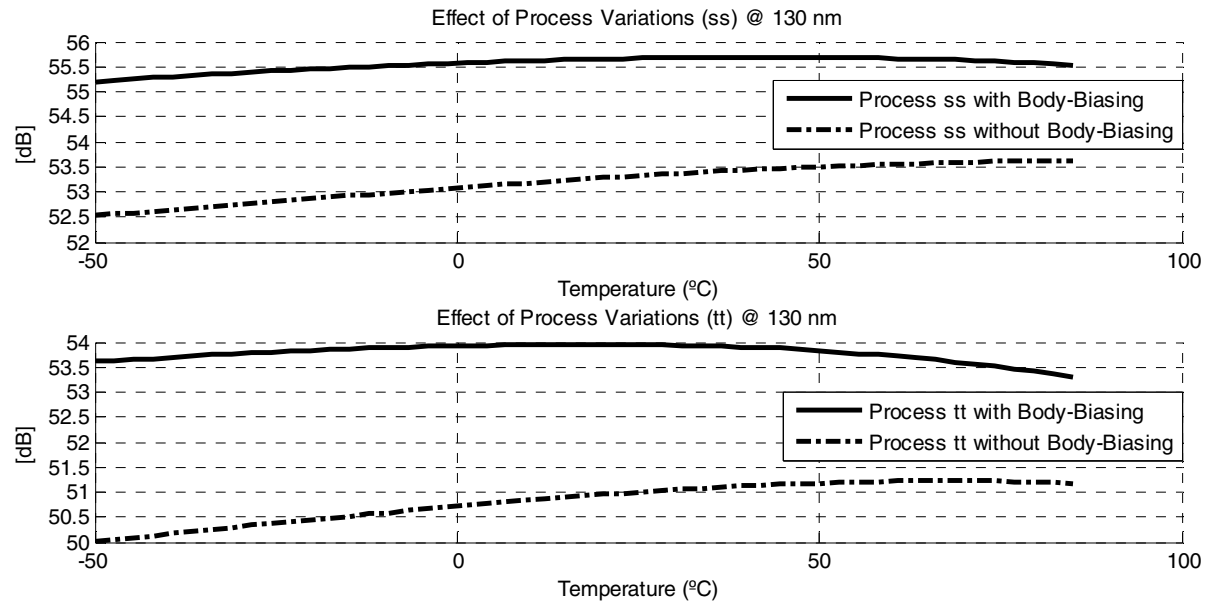

Effect of Process Variations (ff) @ 130 nm

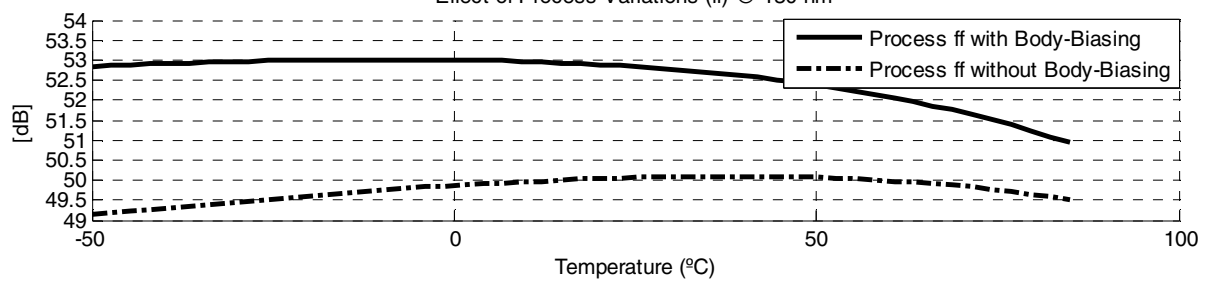

Fig. 10. Overall gain variability with temperature \& process variation for $130 \mathrm{~nm}$ (with and without body-biasing)

\section{Conclusions}

This paper presented a simple circuit technique to reduce gain variability with temperature, supply, and process variations in cascode amplifiers, using a bodybiasing scheme, and at the same time, enhance the overall gain of the amplifier.

Simulation results of a standard telescopic-cascode amplifier, in two different nanoscale CMOS technologies $(130 \mathrm{~nm}$ and $65 \mathrm{~nm}$ ) have shown that it is possible to obtain process-supply-and-temperature-compensation.

The simulated DC gain has low variability (below $\pm 0.5 \mathrm{~dB}$ for all supply-andtemperature variations) while it is enhanced, over all PVT corners by $3 \mathrm{~dB}$.

The authors are unaware of the existence of similar body-biasing compensation schemes to reduce the PVT variability of the DC gain, up to this date. Therefore, it was impossible to perform a comparison with other body-biasing schemes. 


\section{References}

1. Oliveira, J.P., Goes, J.: Parametric Analog Signal Amplification Applied to Nanoscale CMOS Technologies, 2012th edn. Springer (2012)

2. Pekarik, J., Greenberg, D., Jagannathan, B., Groves, R., Jones, J.R., Singh, R., Chinthakindi, A., Wang, X., Breitwisch, M., Coolbaugh, D., Cottrell, P., Florkey, J., Freeman, G., Krishnasamy, R.: RFCMOS technology from $0.25 \mu \mathrm{m}$ to $65 \mathrm{~nm}$ : the state of the art. In: Proc. of the IEEE Custom Integrated Circuits Conf., pp. 217-224 (October 2004)

3. Weste, N.H.E., Eshraghian, K.: Principles of CMOS VLSI Design, 2nd edn. Addison Wesley (1994)

4. Carusone, T.C., Johns, D.A., Martin, K.W.: Analog Integrated Circuit Design, 2nd edn. Wiley (2011)

5. Razavi, B.: Design of Analog CMOS Integrated Circuits, 1st edn. McGraw-Hill Science/Engineering/Math (2000) 